\title{
Pre-exposure Treatment of Cats with Anti-FHV-1 and Anti-FCV Mouse-Cat Chimeric Antibodies
}

\author{
Misako UMEHASHI ${ }^{1,2)}$, Takashi IMAMURA ${ }^{1)}$, Shunsuke AKIYAMA ${ }^{1)}$, Junichi MATSUDA ${ }^{1)}$, Sachio TOKIYOSHI ${ }^{1)}$, \\ Yukinobu TOHYA ${ }^{3)}$ and Takeshi MIKAMI ${ }^{4}$ \\ ${ }^{1)}$ The Chemo-Sero-Therapeutic Research Institute, Kyokushi Kikuchi, Kumamoto 869-1298, ${ }^{2)}$ Kumamoto Health Science University, 325 \\ Izumimachi, Kumamoto 861-5598, ${ }^{3)}$ Graduate School of Agricultural and Life Science, The University of Tokyo, 1-1-1 Yayoi, Tokyo \\ 113-0032 and ${ }^{4}$ College of Bioresource Sciences, Nihon University, 1966 Kameino, Fujisawa, Kanagawa 252-8510, Japan
}

(Received 31 October 2002/Accepted 17 January 2003)

ABSTRACT. Prior to pre-exposure treatment of cats with two mouse-cat chimeric antibodies, FJH2 and F1D7, having neutralizing activity to feline herpesvirus-1 (FHV-1) and cat calicivirus (FCV), respectively, these chimeric antibodies were labeled with ${ }^{125} \mathrm{I}$ and administered to cats to examine their blood kinetics. Concentrations of the both administered chimeric antibodies in the blood reached maximum at the 48th hour post-administration, and the level was 34\% for FJH2 and 54\% for F1D7. Then the concentration levels declined gently, and decreased afterwards to $8.2 \%$ for $\mathrm{FJH} 2$ and $25 \%$ for F1D7 on the 20th day post-administration. The blood half-lives of FJH2 and F1D7 were 8.3 days and 10.7 days, respectively. In order to examine effectiveness in pre-exposure treatment of cats with these chimeric antibodies, cats were administered on the 15th day prior to the challenge infections with FHV-1 and FCV by subcutaneous route with $0.5 \mathrm{~m} l / \mathrm{kg}$ of an FJH-F1D7 mixture being adjusted to contain each chimeric antibody of $10 \mathrm{mg} / \mathrm{m} l$. The cats that received the pre-exposure treatment with the cocktail, showed obvious reductions in manifestations of symptoms caused by those viral infections. The protective effectiveness of the pre-exposure treatment against these viral challenge infections was almost equal to that of the tre atment given at right after these challenge infections.

KEY WORDS: blood half-life, feline calicivirus, feline herpesvirus-1, mouse-cat chimeric antibody, pre-exposure treatment.

J. Vet. Med. Sci. 65(5): 563-566, 2003

Blood half-life of antibodies is one of the important factors giving greater influences on their treatment effects. Weak points of mouse monoclonal antibodies in their application for the treatment of other animal species are induction of immune responses to the mouse antibodies as foreign proteins and that their half-lives are short in the blood. In order to overcome such weak points of mouse monoclonal antibodies, we applied a chimeric antibody strategy [3] and developed two mouse-cat chimeric antibodies, FJH2 and F1D7, against FHV-1 and FCV, respectively [6, 7]. We reported previously that repeated administrations of cats with a mouse-cat chimeric antibody did not induce any anaphylactic reactions, whereas repeated administrations of mouse monoclonal antibody did [6]. We further reported the treatment effects of cats with FJH2 and F1D7 against FHV-1 and FCV infections [7]. In the present study, we will describe the transitions and half-lives of FJH2 and F1D7 in cat blood, and also report protective effect against the dual infections of FHV-1 and FCV in the pre-exposure treatment of cats with those chimeric antibodies.

\section{MATERIALS AND METHODS}

Test materials: Anti-FCV mouse-cat chimeric antibody (F1D7) and anti-FHV-1 mouse-cat chimeric antibody (FJH2) were used for the present studies. The variable region genes used for the construction of F1D7 are originated from those of an anti-FCV neutralizing mouse monoclonal antibody (1D7) that is known to have a wide neutralizing spectrum against a variety of FCV strains in the field [5]. The variable region genes used for the construction of FJH2 are originated from those of a anti-FHV-1 neutralizing mouse monoclonal antibody (JH2 ) [7].

Radio-labeling: Anti-FHV-1 mouse-cat chimeric antibody (FJH2) and anti-FCV mouse-cat chimeric antibody (F1D7) were labeled with ${ }^{125} \mathrm{I}$ using $\mathrm{Na}^{125} \mathrm{I}$ (Pierce Biotechnology, Inc., USA) by IODO-GEN method [2]. Purity of ${ }^{125}$ I-labeled FJH2 ( $\left.{ }^{125} \mathrm{I}-\mathrm{FJH} 2\right)$ or F1D7 $\left({ }^{125} \mathrm{I}-\mathrm{F} 1 \mathrm{D} 7\right)$ was $99.6 \%$ and $99.9 \%$, respectively, and these radio-activities were $11.5 \times 10^{6} \mathrm{cpm} / \mathrm{m} l$ for ${ }^{125} \mathrm{I}-\mathrm{FJH} 2$ and $4.7 \times 10^{6} \mathrm{cpm} / \mathrm{m} l$ for ${ }^{125} \mathrm{I}-\mathrm{F} 1 \mathrm{D} 7$. To know whether cracking of the labeled materials occurred or not in the ${ }^{125}$ I-labelling stages, those ${ }^{125}$ I-labeled preparations were examined by SDS-PAGE, and found to be no cracking band of both ${ }^{125} \mathrm{I}$ labeled chimeric antibodies.

Blood kinetics: Groups of 3 specific pathogen free (SPF) cats (17 week old, 1.4-1.9 kg) purchased from Harlan World Headguarters (Indianapolis, U.S.A.) were administered subcutaneously with $7.5 \mathrm{mg} / \mathrm{kg}$ of ${ }^{125} \mathrm{I}-\mathrm{FJH} 2$ or ${ }^{125} \mathrm{I}-$ F1D7. Subsequently, blood samples were taken from the neck vein into heparin-treated injector on 1, 3, 24, 48, 72, $96,120,144,192,240,288,336,384,432$ and 480th hr post-administration. Assay for ${ }^{125}$ I-radio activity of the blood samples was carried out immediately after their samplings. Count rates were plotted against time in a semi-logarithmic plot, and then half-lives in the blood of those labeled antibodies were extrapolated according to the method of Yamaoka and Tanigawa [8].

Virus neutralization tests: Serum samples for virus neutralization tests were taken on right before the administra- 
tion of FJH2 and F1D7 and on right before the challenge exposures of FHV-1 and FCV. The serum samples were allowed to stand in a water bath at $56^{\circ} \mathrm{C}$ for $30 \mathrm{~min}$ for inactivation, then kept at $-20^{\circ} \mathrm{C}$ until use. Virus neutralization tests were performed according to the method described by Umehashi et al. [7].

Protective effect: $\mathrm{FJH} 2$ and F1D7 were mixed together to contain each antibody of $20 \mathrm{mg}$ per $\mathrm{ml}$, and added with a stabilizer. One $\mathrm{m} l$ of the mixture was distributed into vials, and then lyophilized. The lyophilized product containing $20 \mathrm{mg}$ of FJH2 and F1D7 per vial was reconstituted with $2 \mathrm{~m} l$ of injectable water before use.

Nine SPF cats were introduced from Liberty Research Inc. (Waverly, U.S.A.), and distributed randomly into three groups. Weights of the cats at the time of experiments were $2.55-2.95 \mathrm{~kg}$. Animals were infected by the nasal route simultaneously with $10^{5.0} \mathrm{TCID}_{50}$ of FHV-1 K1 strain and $10^{7.0} \mathrm{TCID}_{50}$ of FCV C-14 strain. On 15 th day prior to infection or right after infection, each group was administered subcutaneously with $0.5 \mathrm{ml} / \mathrm{kg}$ of the mixture of those chimeric antibodies $(5 \mathrm{mg} / \mathrm{kg}$ of $\mathrm{FJH} 2$ and $5 \mathrm{mg} / \mathrm{kg}$ of FJH2). The control group animals were also administered with 0.5 $\mathrm{m} l / \mathrm{kg}$ of physiological saline. Various items including the clinical symptoms listed in Table 1 were monitored until the 20th day post-infection. All the scores with regard to each subject were summed, and calculated the averaged value in each cat group.

Through the present study, all the cats were kept in a well-controlled atmosphere of $55 \pm 5 \%$ moisture and $24 \pm$ $1^{\circ} \mathrm{C}$ for easing their stress, and after the present study they were euthanized by blood letting under general anesthesia.

\section{RESULTS}

Blood kinetics and half-life: Blood concentration of ${ }^{125} \mathrm{I}-$ FJH2 or ${ }^{125}$ I-F1D7 showed a peak at 48th hour post-administration, and the rates of ${ }^{125} \mathrm{I}-\mathrm{FJH} 2$ and ${ }^{125} \mathrm{I}-\mathrm{F} 1 \mathrm{D} 7$ were $34 \%$ and $54 \%$ out of the totally administered antibodies, respectively. Then, the level in the blood of those ${ }^{125}$ I-labeled chimeric antibodies were declined gradually, and the rates on 20th day post-administration were $8.2 \%$ for ${ }^{125} \mathrm{I}-\mathrm{FJH} 2$ and $15.7 \%$ for ${ }^{125} \mathrm{I}-\mathrm{F} 1 \mathrm{D} 7$ (Fig. 1). The half-lives of ${ }^{125} \mathrm{I}-\mathrm{FJH} 2$ and ${ }^{125} \mathrm{I}-\mathrm{F} 1 \mathrm{D} 7$ in the blood were 8.3 and 10.7 days, respectively (Table 2).

Neutralizing antibody titer and protective effect: Neither anti-FHV-1 neutralizing antibody titer nor anti-FCV neutralizing antibody titer was detected in the serum samples taken prior to the administration of FJH2-F1D7 mixture. Neutralizing antibody titers of the serum samples taken on 15th day post-administration were 1: $2-<2$ for anti- FHV-1 and 1: 4-2 for anti-FCV, respectively.

The animals of the control group began to emerge clinical symptoms on 3rd day post-infection, and the onset reached a peak on 7 th day post-infection. In contrast to this, the animals of the groups that were administered FJH2-F1D7 mixture on right after the dual challenge infections showed obviously reduced appearance of symptoms (Fig. 2). Furthermore, the animals that were administered FJH2-F1D7

Table 1. Standard of observation

\begin{tabular}{|c|c|c|c|c|c|}
\hline \multirow{2}{*}{ Item } & \multicolumn{5}{|c|}{ Score } \\
\hline & 0 & 1 & 2 & 3 & 4 \\
\hline Activity & normal & disappear a little & disappear & & \\
\hline Appetite $^{a)}$ & $\geq 90 \mathrm{~g}$ & $50 \mathrm{~g}-90 \mathrm{~g}$ & $<50 \mathrm{~g}$ & & \\
\hline Drinking ${ }^{\mathrm{b})}$ & $\geq 125 \mathrm{ml}$ & $50 \mathrm{~m} l-125 \mathrm{~m} l$ & $<50 \mathrm{~m} l$ & & \\
\hline Feces & normal & soft & diarrhea & & \\
\hline Vomiting & - & & + & & \\
\hline General condition & normal & sensitive/piloerection & trembling & & \\
\hline Dehydration $^{c)}$ & promptly & $2-5 \mathrm{sec}$ & $6-20 \mathrm{sec}$ & $\geq 21 \mathrm{sec}$ & \\
\hline Sneezing ${ }^{\mathrm{d})}$ & 0 & $1-4$ times & $5-9$ times & $\geq 10$ times & \\
\hline Sialorrhea & - & a little & much & purulent & \\
\hline Ophthalmia & - & conjunctivitis & purulent conjunctivitis & corneal ulcer & \\
\hline Rhinorrhea & - & watery (a little) & watery (much) & purulent & \\
\hline Muzzle ulcer & - & bulla/reddening & skin deficit & ulcer & erosive ulcer \\
\hline Stomatitis ${ }^{\mathrm{e}}$ & - & bulla/reddening & ulcer ( $\geq 2$ places) & ulcer ( $\leq 3$ places) & erosive ulcer \\
\hline Respiration & normal & nasal breathing & dyspnea & two-phase & \\
\hline
\end{tabular}




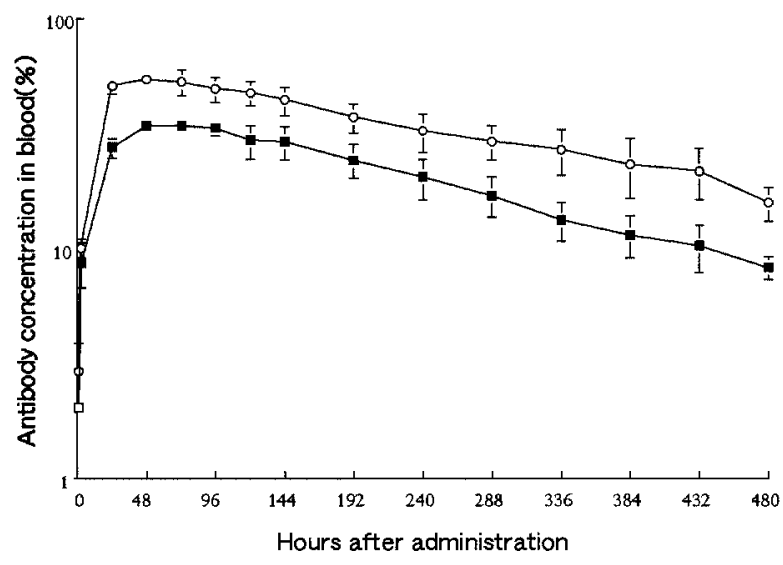

Fig. 1. Concentration of ${ }^{125} \mathrm{I}-\mathrm{FJH} 2$ and ${ }^{125} \mathrm{I}-\mathrm{F} 1 \mathrm{D} 7$ in blood. The SPF cats were subcutaneously administered $7.5 \mathrm{mg} / \mathrm{kg}$ of ${ }^{125} \mathrm{I}-$ FJH2 or ${ }^{125}$ I-F1D7 into the back. Subsequently blood samples were taken from the neck vein on 1, 3, 24, 48, 72, 96, 120, 144, $192,240,288,336,384,432$ and 480th hr post-administration, and assayed for radioiodine activity $(\mathrm{n}=3)$. FJH2 administration (-ם-), F1D7 administration (- - -).

Table 2. Blood half-life of ${ }^{125}$ I-FJH2 and ${ }^{125}$ I-F1D7

\begin{tabular}{cccc}
\hline & cat No & $\begin{array}{c}\text { half-life } \\
\text { (hours) }\end{array}$ & $\begin{array}{c}\text { average } \\
\text { (hours) }\end{array}$ \\
\hline \multirow{3}{*}{ FJH2 } & 1 & 7.7 & \\
& 2 & 8.3 & 8.3 \\
& 3 & 8.8 & \\
F1D7 & 1 & 9.3 & \\
& 2 & 10.4 & 10.7 \\
& 3 & 12.5 & \\
\hline
\end{tabular}

mixture on 15th day prior to the dual challenge infection also showed obviously reduced appearance of symptoms. Protective effect of the pre-exposure treatment was almost the same as that of the treatment at right after infection (Fig. 2).

\section{DISCUSSION}

When mouse monoclonal antibodies are used for treatment of infection in other animal species including humans, there remains some problems such as shorten half-lives in the blood, lower effector functions and induction of antimouse antibodies. To overcome these weak points there have been reported chimeric or reshaped antibody strategies [4].

In the present study, we examined the half-lives of FJH2 and F1D7 in the cat blood. The half-lives of FJH2 and F1D7 were 8.3 and 10.7 days, respectively. It is interesting that the half-life of F1D7 was longer than that of FJH2 by approximately 2 days despite a common $\mathrm{C}$-region gene (CB25 $\gamma$ ) was used for the constructions of those chimeric antibodies. It is considered that the difference in the blood half-lives of FJH2 and F1D7 can be attributed to the differ-

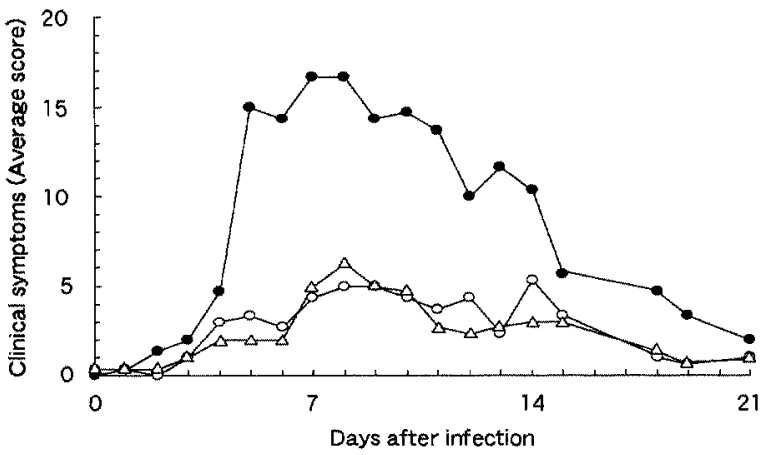

Fig. 2. Protective effect of chimeric antibody. The SPF cats were infected intranasally with $10^{5.0} \mathrm{TCID}_{50}$ of FHV-1 and $10^{7.0}$ $\mathrm{TCID}_{50}$ of FCV. On the 15 th day before the infection or right after infection, 3 animals in each group were administered subcutaneously $0.5 \mathrm{~m} l / \mathrm{kg}$ of chimeric antibody mixture $(5 \mathrm{mg} / \mathrm{kg}$ of FJH2 and $5 \mathrm{mg} / \mathrm{kg}$ of F1D7). The control group animals were also administered $5 \mathrm{~m} l / \mathrm{kg}$ of physiological saline. Prior to infection and until the 21 st day after infection, the clinical symptoms was monitored, and the appearance of symptoms was scored. Administrated right after infection (- $\bigcirc$-), Administered on the 15th day before the infection $\left(-\triangle_{-}\right)$, Physiological salineadministered group (-)

ence between the V-regions of them. Unfortunately, there has been no report on half-life in the cat blood of mouse monoclonal antibodies including 1D7 and JH2 that are the parental mouse antibodies corresponding to F1D7 and FJH2, respectively. Regarding the half-life of mouse antibodies in the human blood, it was reported as 0.6-0.7 days, but those of the corresponding mouse-human chimeric antibodies were prolonged to 3-6 days [4]. To know the prolonging effect on the blood half-life of the present chimerization where mouse C-region was substituted to cat one, further studies on the parental mouse antibodies are essential. Studies on the half-lifives of those chimeric antibodies based on their neutralizing activities will be also important, for trace by neutralizing activity means that of intact antibody or at least functional one. However, in the present study, we used ${ }^{125}$ I-laveled antibodies and did not use those neutralization tests due to their low sensitivity.

In the present pre-exposure treatment study, we applied the infection model with SPF cats that was established for evaluation of the protective efficacy of FJH2 and F1D7 [7]. Although detailed data were not shown in the present study, the relationships between viral infections and clinical manifestations in the animal infection model have been clarified. Namely, clinical manifestations of the animals infected with FHV-1 or FCV are very characteristic. For example, stomatitis is mainly manifested in the FCV infection, but it is rare and other manifestations such as salivation, conjunctival edema, spasmodic cough, secretion/excretion from eyes/ nose and so on are strongly manifested in the FHV-1 infection. We administered those chimeric antibodies on 15th day prior to the FHV-1-FCV dual infections. Regarding the peak concentrations of those chimeric antibodies in the cat 
blood as 1.0, and also as the half-lives of FJH2 and F1D7 were approximately 8 and 12 days, respectively, the blood concentrations on 15th day post-administration can be estimated as 0.3 for $\mathrm{FJH} 2$ and 0.4 for F1D7. In the present preexposure study, anti-FHV-1 and anti FCV neutralizing antibody titers in the serum samples taken on 15 th day postadministration were $1: 2-<2$ and 1:4-2, respectively. In the present study, administration of those chimeric antibodies on 15th day prior to the exposures to FHV-1 and FCV infections was as effective as that on right after those viral exposures. It is known that there are some serotypes for FCV in the field [1], and neutralizing spectrum of anti-FCV antibody to use for the treatment of FCV-infected cats will be one of the most important issues. Fortunately, the neutralizing spectrum of 1D7 as the parental mouse monoclonal antibody of F1D7 is relatively wide [5]. Regarding FHV-1, there have been no report on such issue of serotypes. From the serological properties of F1D7 and FJH2 and the results in the present studies, we concluded it is possible to use those chimeric antibodies for not only therapeutic purpose but also prophylactic one.

ACKNOWLEDGEMENT. The authors thank Miss Yoko Tomita (The Chemo-Sero-Therapeutic Research Institute) for her supports on the present study.

\section{REFERENCES}

1. Kalunda, M., Lee, K.M., Holmes, D.F. and Gillespie, J.H. 1975. Serologic classification of feline caliciviruses by plaquereduction neutralization and immunodeffusion. Am. J. Vet. Res. 36: 353-356.

2. Millar, W.T. and Smith, J.F. 1983. Protein iodination using Iodogen. Int. J. Appl. Radiat. Isot. 34: 639-641.

3. Morrison, S.L., Jhonson, M.J., Herzenberg, L.A. and Oi, V.T. 1984. Chimeric human antibody molecules;Mouse antigenbinding domains with human constant region domains. Proc. Natl. Acad. Sci. U.S.A. 81: 6851-6855.

4. Noguchi, H. 1993. Principle and clinical application of chimeric antibody/humanized antibody. J. Clin. Exp. Med. 167: 457-462.

5. Tohya, Y., Masuoka, K., Takahashi, E. and Mikami, T. 1991 Neutralizing epitopes of feline calicivirus. Arch Virol. 117: 173-181.

6. Umehashi, M., Imamura, T., Akiyama, S. and Tokiyoshi, S 2002. Development and safety of a mouse-cat chimeric antibody against the feline calicivirus. J. Jpn. Vet. Med. Assoc. 55: 293-297.

7. Umehashi, M., Imamura, T., Akiyama, S., Kimachi, K., Tokiyoshi, S. and Mikami, T. 2002. Post-exposure treatment of cats with mouse-cat chimeric antibodies against feline herpesvirus type 1 and feline calicivirus. J. Vet. Med. Sci. 64: 1017-1021.

8. Yamaoka, K. and Tanigawara, Y. 1983. Automatic blood concentration data analysis program. pp. 159-178. In: A Pharmacokinetics Guide with a Micro Computer. Nankodo, Tokyo (in Japanese). 